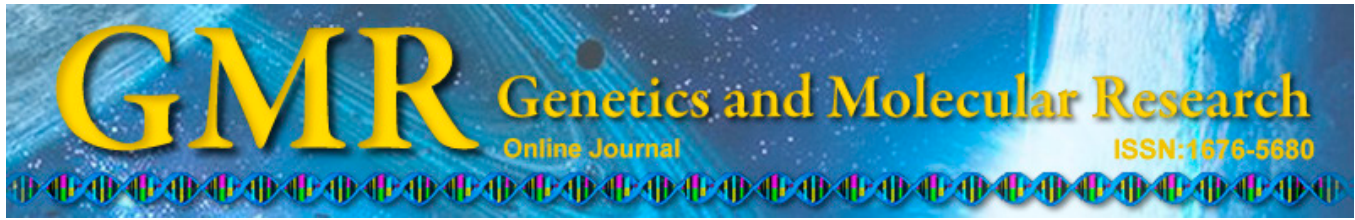

\title{
Association of SIRT2 gene polymorphisms with body measurement and growth traits of Qinchuan cattle
}

\author{
L.S. Gui ${ }^{1}$, W.C. Yang ${ }^{1,2}$, C.P. Zhao ${ }^{1,2}$, S.J. Wei ${ }^{1}$, Z.D. Zhao ${ }^{1}$ and L.S. Zan ${ }^{1,2}$ \\ ${ }^{1}$ College of Animal Science and Technology, Northwest A\&F University, \\ Yangling, Shaanxi China \\ ${ }^{2}$ National Beef Cattle Improvement Center of Northwest A\&F University, \\ Yangling, Shaanxi China
}

Corresponding author: L.S. Zan

E-mail: zanlinsen@163.com

Genet. Mol. Res. 13 (4): 8834-8844 (2014)

Received October 16, 2013

Accepted March 25, 2014

Published October 27, 2014

DOI http://dx.doi.org/10.4238/2014.October.27.24

\begin{abstract}
Silent information regulator 2 (SIRT2), a member of the Sirtuin family of class III nicotinamide adenine dinucleotidedependent protein deacetylases, plays an important role in senescence, metabolism, and apoptosis. This study was conducted to detect potential polymorphisms of the bovine SIRT2 gene and explore their relationships with meat quality and body measurement traits (BMTs) in Qinchuan cattle. Four single nucleotide polymorphisms (A7445G, C7711T, G17937A, and G20937A) in the fourth intron, fourth exon, ninth exon, and twelfth exon of the SIRT2 gene, respectively, were identified according to the sequencing results of 520 individuals of a Qinchuan cattle population. The genotypic distributions of both A7445G and G20937A were in agreement with the Hardy-Weinberg equilibrium $(\mathrm{P}<0.05)$, whereas the other two mutations were not $(0.05<\mathrm{P}<0.01)$, based on the $\chi^{2}$ test. Association analysis indicated that the four loci were significantly correlated with several BMTs and meat quality traits. When in combination, the $\mathrm{H}_{1} \mathrm{H}_{1}$ (AA-CC-GG-CC) diplotypes showed better BMT and meat quality traits than those by other combinations.
\end{abstract}


Collectively, the results show that SIRT2 is involved in the regulation of the growth and meat quality of cattle, suggesting that the SIRT2 gene may be a candidate gene for marker-assisted selection in the development of future breeding programs for Qinchuan cattle.

Key words: Genetic variability; SIRT2; Body measurement; Meat quality; Single nucleotide polymorphism

\section{INTRODUCTION}

Chinese indigenous yellow cattle are known to have a strong trunk, high stress resistance, and good environmental adaptability. However, drawbacks of this breed remain, including their underdeveloped hind hips and slow growth rates. Therefore, it is necessary to select important functional genes of beef cattle through marker-assisted selection in order to increase their productivity, and thereby economic benefits, and promote the development of the Chinese cattle industry toward high quality and efficiency.

In mammals, homologs of silent information regulator 2 (SIRT2) are named sirtuins, and belong to the class III nicotinamide adenine dinucleotide (NAD)-dependent deacetylase family (Guarente, 2007). Seven sirtuin members, designated as SIRT1-SIRT7, have been identified to date. Among them, SIRT2, a tubulin deacetylase, is localized mainly in the cytoplasm and plays either detrimental or beneficial roles in cell survival under different conditions (Zhou et al., 2012). The SIRT2 gene was found to be ubiquitously expressed as NAD ${ }^{+}$-dependent protein deacetylases (Peck et al., 2010), and plays critical roles in a variety of different biological processes, such as longevity and metabolism, through the deacetylation of histones (Blander and Guarente, 2004; Marmorstein, 2004).

Recent studies have suggested that the SIRT2 gene could enhance the proliferation of preadipocytes and inhibit preadipocyte apoptosis. SIRT2 could induce FOXO1 gene deacetylation in rats (Wang and Tong, 2009). Overexpression of SIRT2 caused FoxO1 acetylation/ deacetylation and reduced the expression of PPAR $\gamma$ and $\mathrm{C} / \mathrm{EBP} \alpha$ in 3T3-L1 cells, and these changes led to abnormal mitochondrial morphology and inhibited adipogenesis (Jing et al., 2007). Moreover, transcriptional repression of SIRT2 resulted in inhibition of fatty acid oxidation and energetic uncoupling via hypoxia-inducible factor $1 \alpha$ accumulation in diabetic humans and mice (Krishnan et al., 2012). Based on the deduced biological function of SIRT2 in humans and mice, we hypothesized that the SIRT2 gene might be associated with cattle body measurement and meat quality traits.

However, polymorphisms of the SIRT2 gene have not yet been reported for cattle. Therefore, this study aimed to determine the SIRT2 gene function using bioinformatics information, and its tissue expression pattern was analyzed using real-time polymerase chain reaction (PCR). In addition, we sought to identify quantitative trait loci relevant to growth and meat quality traits in Qinchuan cattle.

\section{MATERIAL AND METHODS}

\section{Genomic DNA preparation and phenotypic data collection}

A total of 520 unrelated adult animals were randomly selected from Qinchuan cattle 
breeding populations, which ranged in age from 18 to 24 months. DNA samples were extracted from blood samples collected from the jugular vein and stored at $-80^{\circ} \mathrm{C}$ according to the standard phenol chloroform protocol (Sambrook and Russell, 2001). The DNA content was estimated spectrophotometrically, and then the genomic DNA was diluted to $50 \mathrm{ng} / \mathrm{L}$. All DNA samples were stored at $-20^{\circ} \mathrm{C}$ for subsequent analysis.

Body measurement traits (BMTs), including body length (BL), withers height (WH), hip height (HH), rump length (RL), hip width (HW), chest depth (CD), chest circumference (CC), and pin bone width (PBW), were measured as described previously (Gilbert et al., 1993). The meat quality traits, including backfat thickness (BT), ultrasound loin muscle area (ULA), and intramuscular fat (IF), were measured using the Rincon method (Rincon et al., 2009). In order to reduce systematic error, a single investigator was assigned to measure 1 of the 11 traits in all animals.

\section{Primer design and PCR conditions}

Based on the bovine SIRT2 gene sequence (GenBank accession No. NM_001113531.1), three pairs of PCR primers were designed to amplify different fragments of the SIRT2 gene. Primers, annealing temperature, and fragment sizes are given in Table 1. The PCR amplification product was amplified from a $20-\mu \mathrm{L}$ mixture comprising $50 \mathrm{ng}$ DNA, $10 \mathrm{pM}$ of each primer, $0.20 \mathrm{mM}$ dNTP, $2.5 \mathrm{mM} \mathrm{MgCl}$, and 0.5 U Taq DNA polymerase (TaKaRa, Dalian, China). Amplification was programmed for an initial $5 \mathrm{~min}$ at $95^{\circ} \mathrm{C}$, followed by 35 cycles of $94^{\circ} \mathrm{C}$ for $30 \mathrm{~s}$, annealing temperature of $63.3^{\circ}, 63.3^{\circ}, 58.5^{\circ}$ and $65.5^{\circ} \mathrm{C}$ (for A7445G, C7711T, G17937A, and G20937A, respectively ) for $30 \mathrm{~s}$, and $72^{\circ} \mathrm{C}$ for $30 \mathrm{~s}$, and a final extension at $72^{\circ} \mathrm{C}$ for $10 \mathrm{~min}$. The digested products were detected by electrophoresis on a $1.0 \%$ agarose gel stained with ethidium bromide, purified using Axygen kits (BMI Fermentas, Glen Burnie, MD, USA), and finally sequenced in both directions in an ABI PRISM 377 DNA sequencer (Perkin-Elmer). The sequence maps were analyzed with the SeqMan software (version 10.3).

Table 1. Primers used for polymerase chain reaction amplification of the SIRT2 gene in Qinchuan cattle.

\begin{tabular}{lllc}
\hline Primer & Primer sequence (5' to $\left.3^{\prime}\right)$ & Length, location & Tm $\left({ }^{\circ} \mathrm{C}\right)$ \\
\hline P1 & 1F: 5'-TGTCCTAGAGCCCACACGC-3' & 724 bp/intron 4 and exon 4 & 66.3 \\
& 1R: 5'-GATACTCACTCTCTGCTTGTCC-3' & & \\
P2 & 2F: 5'-GGTTCACTCCTGACCCTC-3' & 408 bp/exon 9 & 58.5 \\
& 2R: 5'-CATGGCCCAACTAAAGAC-3' & 623 bp/exon 12 & 65.5 \\
P3 & 3F: 5'-CTGTCCCCGTGTCTGTCTGT-3' & & \\
& 3R: 5'-CCTGGAATCTGACCCCTGAG-3' & & \\
\hline
\end{tabular}

\section{Genotyping of SIRT2 alleles by sequencing}

The use of single-strand conformation polymorphism to detect four mutations is a time-consuming and complicated process. In addition, there are no suitable restriction endonucleases for restriction fragment length polymorphism, which is another common method for detecting genotype mutant forms of a gene. Therefore, all of the products obtained from the DNA samples of the 520 Qinchuan cattle were directly sequenced for distinguishing the genotypes of the four mutations in SIRT2. 


\section{Statistical analysis}

Gene frequencies, allelic frequencies, and deviations from Hardy-Weinberg equilibrium were determined by direct counting. Population genetic indices including heterozygosity $\left(H_{\mathrm{E}}\right)$, homozygosity $\left(H_{\mathrm{O}}\right)$, effective allele numbers $\left(N_{\mathrm{E}}\right)$, and polymorphism information content (PIC) were calculated according to Nei's methods (Nei and Roychoudhury, 1974). Linkage disequilibrium (LD) and haplotype distributions of the single nucleotide polymorphisms (SNPs) were analyzed using the expectation maximization algorithm, as obtained through the Haploview software (Barrett et al., 2005).

The SPSS software (version 13.0) was used to analyze the relationship between different genotypes of the SIRT2 gene and the BMTs (BL, WH, HH, RL, HW, CD, CC, and PBW) and meat quality traits (BT, ULA, and IF) of Qinchuan cattle. The following statistical linear model was used: $Y i j=\mu+G i+A i+E i j k$, where $Y i j$ is the trait value for each individual, $\mu$ is the overall population mean for the traits, $G i$ is the fixed effect associated with genotype, $A i$ is the fixed effect of age, and Eijk is the standard error.

\section{RESULTS}

\section{Polymorphisms and genetic diversity}

Four polymorphism sites in the SIRT2 gene (Table 1), A7445G (Figure 1), C7711T (Figure 2), G17937A (Figure 3), and G20937A (Figure 4), were identified by sequencing, which were located in intron 4, exon 4, exon 9, and exon 12, respectively. Both G17937A and G20937A are missense mutations (Val221Ile and Val299Ile), whereas C7711T is a nonsense mutation.

The DNA restriction fragments for each SNP are shown in Figures 1-4. G17937A had only two genotypes, as the AA genotype was not detected in the sampled animals. Genotype and allele frequencies for the four loci are shown in Table 2. Allele $\mathrm{G}$ was predominant for G17937A and G20937A, whereas alleles A and C were predominant for A7445G and C7711T, respectively. Results of the $\chi^{2}$ test illustrated that the genotypic distributions of both the A7445G and G20937A mutations were in agreement with Hardy-Weinberg equilibrium ( $P$ $<0.05)$, while the other two mutations were not $(0.05<\mathrm{P}<0.01)$.

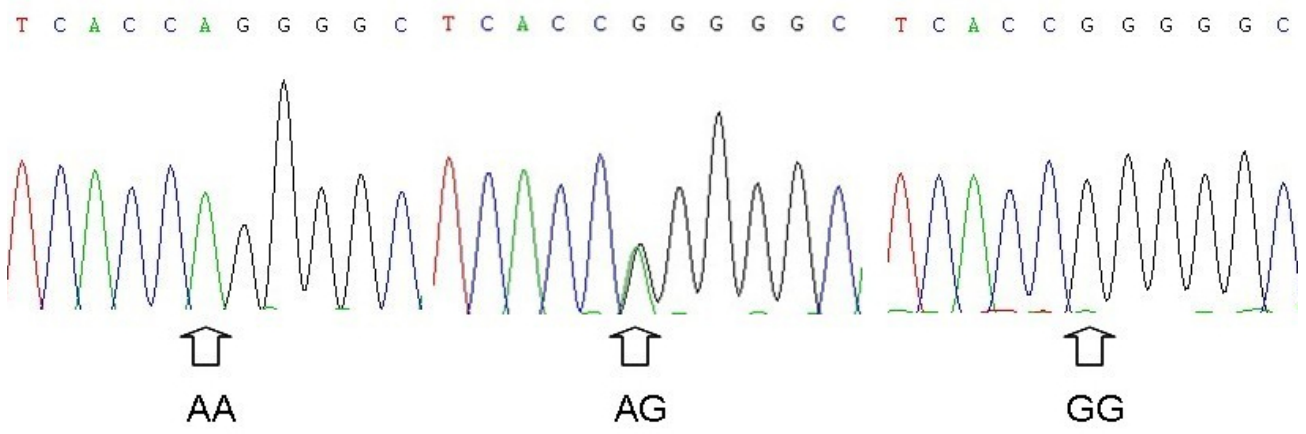

Figure 1. Sequencing map of the snp7445 locus. 


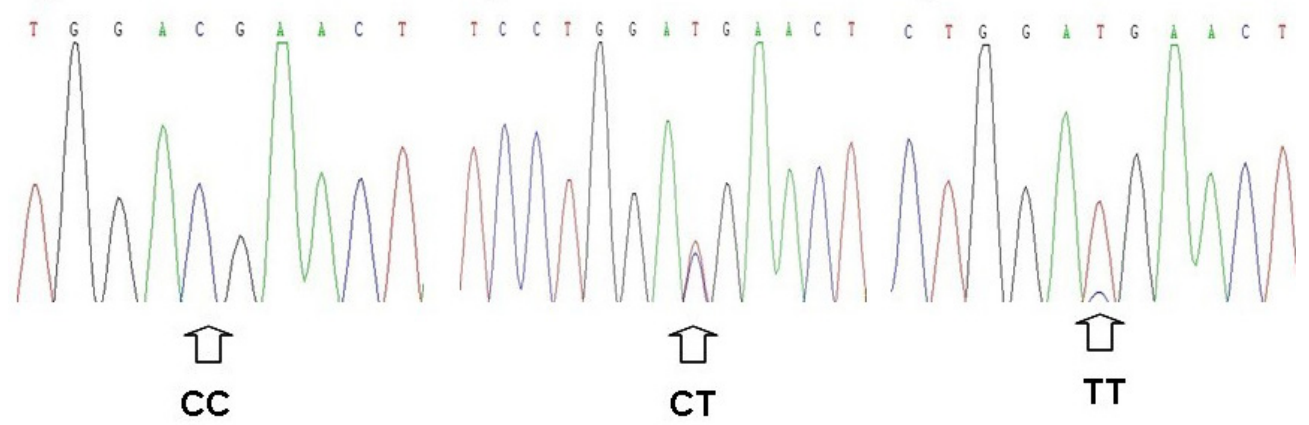

Figure 2. Sequencing map of the snp7711 locus.

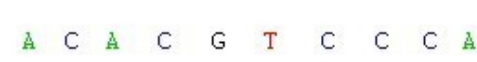
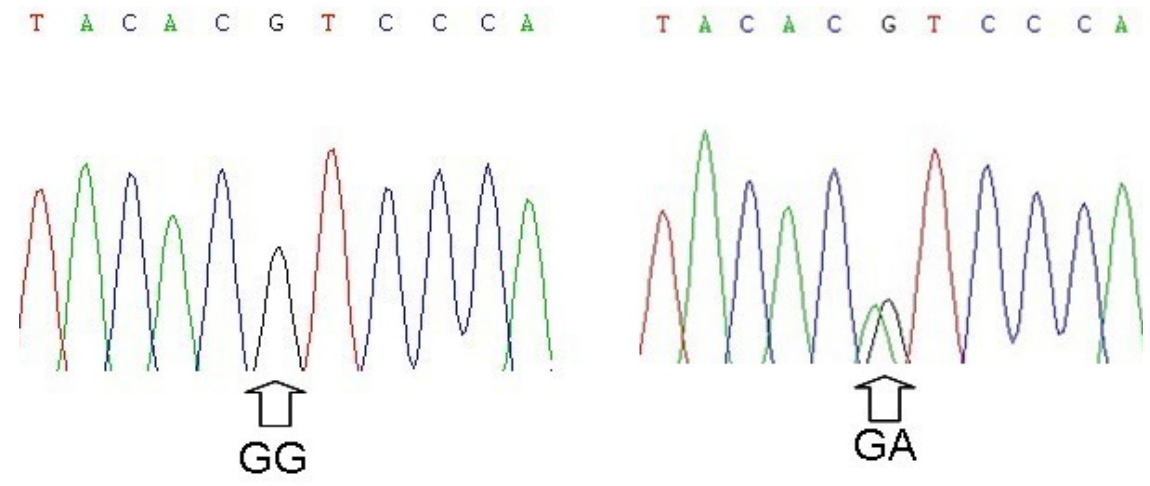

Figure 3. Sequencing map of the snp17937 locus.

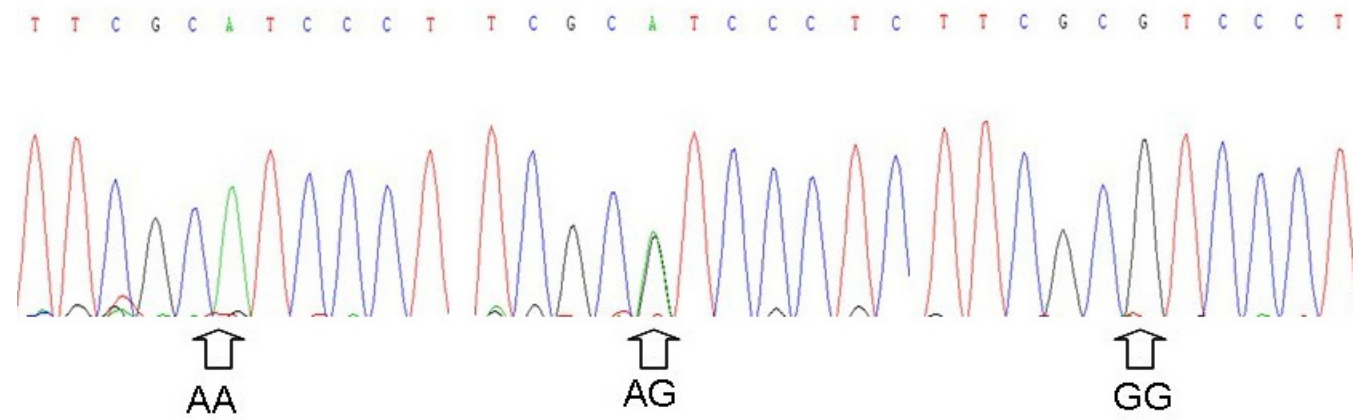

Figure 4. Sequencing map of the snp20937 locus.

Based on Nei and Roychoudhury (1974), the population genetic indices $H_{\mathrm{E}}, N_{\mathrm{E}}$, and PIC were calculated, and the results are shown in Table 2. The SNPs A7445G, C7711T, and G20937A showed a medium polymorphism level $(0.25<$ PIC $<0.50)$, whereas G17937A showed a low polymorphism level (PIC $<0.25$ ). 
Table 2. Genotype frequencies (\%) of the SIRT2 gene for the single nucleotide polymorphisms in the Qinchuan cattle populations.

\begin{tabular}{|c|c|c|c|c|c|c|c|c|c|c|}
\hline \multirow[b]{2}{*}{$\mathrm{A} 7445 \mathrm{G}$} & \multirow{2}{*}{$\begin{array}{c}\text { Sample } \\
520\end{array}$} & \multicolumn{3}{|c|}{ Genotypic frequency } & \multicolumn{2}{|c|}{ Allele frequency } & \multirow{3}{*}{$\begin{array}{c}H_{\mathrm{E}} \\
0.4466\end{array}$} & \multirow{3}{*}{$\frac{N_{\mathrm{E}}}{1.8069}$} & \multirow{3}{*}{$\begin{array}{c}\text { PIC } \\
0.3469\end{array}$} & \multirow{3}{*}{$\begin{array}{c}\chi^{2} \text { (HWE) } \\
4.7577\end{array}$} \\
\hline & & AA & $\mathrm{AG}$ & GG & A & G & & & & \\
\hline & & 0.4615 & 0.4039 & 0.1346 & 0.6635 & 0.3365 & & & & \\
\hline \multirow[t]{2}{*}{ C7711T } & 520 & $\mathrm{CC}$ & $\mathrm{CT}$ & TT & C & $\mathrm{T}$ & & & & \\
\hline & & 0.5731 & 0.3365 & 0.0904 & 0.7413 & 0.2587 & 0.3835 & 1.6221 & 0.3100 & 7.7987 \\
\hline \multirow[t]{2}{*}{ G17937A } & 520 & GG & GA & & G & A & & & & \\
\hline & & 0.8058 & 0.1942 & 0 & 0.9029 & 0.0971 & 0.1754 & 1.2127 & 0.1600 & 6.0161 \\
\hline \multirow[t]{2}{*}{ G20937A } & 520 & GG & GA & AA & G & A & & & & \\
\hline & & 0.5346 & 0.3654 & 0.1000 & 0.7173 & 0.2827 & 0.4056 & 1.6822 & 0.3233 & 5.101 \\
\hline
\end{tabular}

HWE $=$ Hardy-Weinberg equilibrium; $\chi 0.05^{2}=5.991, \chi 0.01^{2}=9.21$. Genotype distributions of the mutation were in agreement with HWE if $\chi^{2}$ (P value) was less than $0.05^{2}(0.05)$, genotype distributions of the mutation were not in HWE if $\chi^{2}$ (P value) was greater than $\chi 0.05^{2}(0.05) . H_{\mathrm{E}}=$ heterozygosity; $N_{\mathrm{E}}=$ effective number of alleles; PIC $=$ polymorphism information content.

\section{LD and haplotype analysis}

LD between polymorphism pairs and haplotype structure analyses of the SIRT2 gene in Qinchuan cattle are shown in Tables 3 and 4. The $r^{2}$ values for LD between the four sites ranged from 0.000 to 1.000 . Ardlie et al. (2002) suggested that if the $r^{2}$ value is $>0.33$, LD is considered to be strong. Our results revealed strong linkage between A7445G and G17937A, as well as between C7711T and G20937A.

The four SNPs identified showed seven different haplotypes in the populations studied (frequency $>0.05$ ). Hap1 (-ACGC-) had the highest haplotype frequency $(27.70 \%)$, followed by Hap2 (-ACGG-) and Hap7 (-GTGC-), 21.9 and 13.3\%, respectively.

Table 3. Estimated values of linkage disequilibrium for single nucleotide polymorphisms of the bovine SIRT2 gene in Qinchuan cattle.

\begin{tabular}{lcccccc}
\hline $\mathrm{z}$ & A7445G-G17937A & A7445G-C7711T & A7445G-G20937A & G17937A-C7711T & G17937A-G20937A & C7711T-G20937A \\
\hline $\mathrm{r}^{2}$ & 0.056 & 0.001 & 0.028 & 0.000 & 0.103 & 0.041 \\
\hline
\end{tabular}

Table 4. Haplotypes of the SIRT2 gene and their frequencies in Qinchuan cattle.
\begin{tabular}{lccccc}
\hline Haplotype & A7445G & C7711T & G17937A & G20937A & Frequency \\
\hline Hap1 & A & C & G & C & 0.277 \\
Hap2 & A & C & G & G & 0.219 \\
Hap3 & A & C & A & C & 0.049 \\
Hap4 & A & T & G & C & 0.092 \\
Hap5 & G & C & G & G & 0.121 \\
Hap6 & G & C & G & C & 0.054 \\
Hap7 & G & T & G & & 0.133 \\
\hline
\end{tabular}

\section{Effects of single markers/haplotype combinations on growth traits and meat quality traits in Qinchuan cattle}

Table 5 summarizes the effects of the SNPs on growth performance and meat quality traits in Qinchuan cattle. At the 7445 SNP locus, individuals with genotype AA had higher values of $\mathrm{BL}, \mathrm{HH}, \mathrm{HW}$, and $\mathrm{CD}$ compared to individuals with genotype $\mathrm{GG}(\mathrm{P}<0.01)$. In 


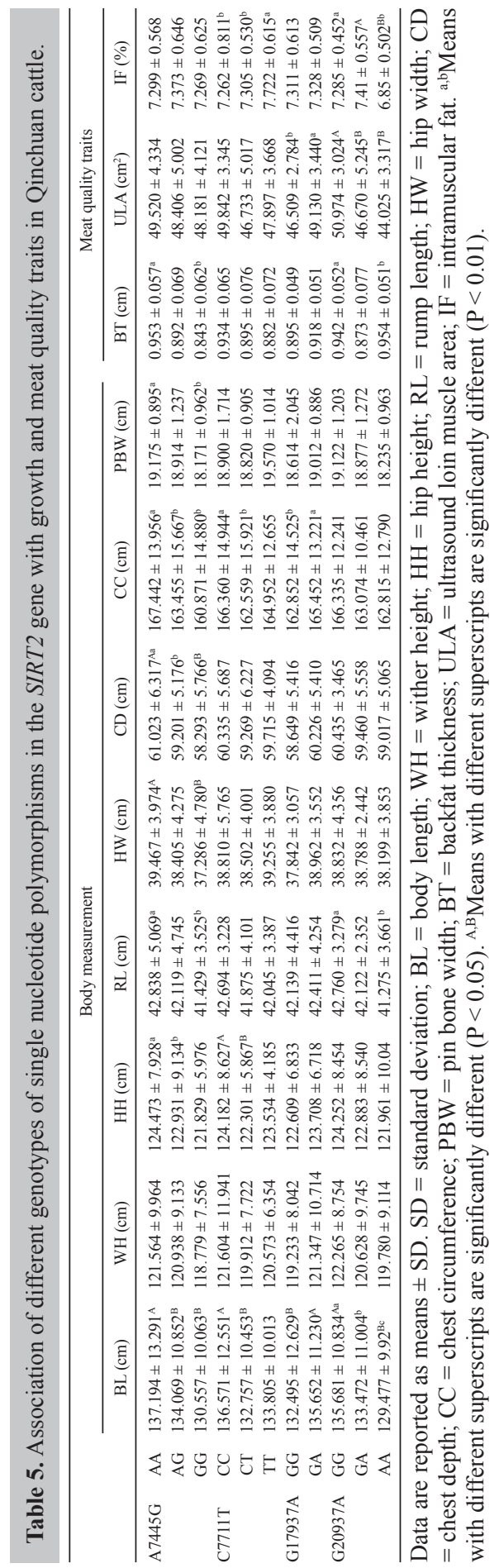


addition, the $\mathrm{CC}, \mathrm{PBW}$, and $\mathrm{BT}$ values were also higher in AA carriers than GG carriers $(\mathrm{P}<$ 0.05). At the 7711 SNP locus, significant differences in BL and $\mathrm{HH}$ were observed between $\mathrm{CC}$ and $\mathrm{CT}$ genotypes $(\mathrm{P}<0.01)$; individuals with the TT genotype had increased IF compared to those with $\mathrm{CC}$ and $\mathrm{CT}(\mathrm{P}<0.05)$. At the $17937 \mathrm{SNP}$ locus, individuals with the AG genotype had significantly higher BL $(\mathrm{P}<0.01), \mathrm{CC}$, and ULA values $(\mathrm{P}<0.05)$ than those with the AA genotype. At the 20937 SNP locus, significant differences of BL and ULA were observed between GG and AA genotypes $(\mathrm{P}<0.01)$, and the RL and BT values of individuals with genotype GG were higher than those of individuals with genotype AA $(\mathrm{P}<0.05)$; compared with AA, individuals with the GA genotype showed increased IF $(\mathrm{P}<0.01)$. However, no significant correlations were observed in the other indices for the four SNPs.

The effects of genetic variations of a gene could be demonstrated more readily by integrating haplotype combination analyses with the single-locus effects. The effects of the combinations of the four SNPs were evaluated, and a total of 14 haplotype combinations were identified; combinations with frequencies lower than 5\% (data not shown) were not included, and the remaining combinations were selected for further analysis. As shown in Table 6, the $\mathrm{H}_{1} \mathrm{H}_{1}$ diplotype had significantly greater $\mathrm{HH}$ values than the $\mathrm{H}_{5} \mathrm{H}_{7}$ diplotype $(\mathrm{P}<0.01)$, and similar results were found for $\mathrm{BL}(\mathrm{P}<0.01)$. In addition, individuals with the combined genotype $\mathrm{H}_{2}-\mathrm{H}_{5}$ had significantly lower growth traits compared to other combinations $(\mathrm{P}<0.01)$. For meat quality traits, $\mathrm{H}_{1} \mathrm{H}_{1}$ and $\mathrm{H}_{4} \mathrm{H}_{7}$ individuals had significantly greater IF values than those with the $\mathrm{H}_{1} \mathrm{H}_{2}$ diplotype $(\mathrm{P}<0.05)$. In addition, association analyses showed highly significant differences between $\mathrm{H}_{2} \mathrm{H}_{5}$ and the other diplotypes $(\mathrm{P}<0.05)$ in $\mathrm{BT}$ and ULA, in which the values of $\mathrm{H}_{2} \mathrm{H}_{5}$ individuals were lowest among all combinations.

\section{DISCUSSION}

In livestock breeding, body measurement and meat quality traits are affected by many factors including genotype, sex, age, breed, herd, location, and other random environmental factors, and are important indices for assessing the economic value of animals (Liu et al., 2010). Through marker-assisted selection studies, many important genes have been identified to be involved in controlling growth (Xue et al., 2011; Tian et al., 2011) and meat quality in livestock (Jiao et al., 2010; Fan et al., 2011). Studies in mammalian cells have suggested that SIRT2 may play a role in cell cycle regulation and cytoskeleton organization by targeting the cytoskeletal protein tubulin (North et al., 2003). Although ample evidence has demonstrated that SIRT2 plays an important role in lipid metabolism and bone growth in humans and mice, its effects on cattle growth and meat quality remain unclear. Interestingly, Jing et al. (2007) found that SIRT2 causes FoxO1 acetylation/deacetylation and reduces the expression of PPAR $\gamma$ and C/EBP $\alpha$ in 3T3-L1 cells, leading to the inhibition of adipogenesis. Moreover, a recent study revealed that SIRT2 suppresses adipocyte differentiation by deacetylating FOXO1 and enhancing its repressive interaction with PPAR $\gamma$ (Wang and Tong, 2009). Moreover, several previous studies have confirmed that SIRT2 plays an important role in the cell cycle, and overexpression of the wild-type SIRT2 gene can prolong the mitotic phase in the cell cycle (Dryden et al., 2003; North and Verdin, 2007; Nahhas et al., 2007). These studies indicate that the SIRT2 gene may mediate, directly or indirectly, meat production traits in animals.

Our results showed that individual cattle with the AA genotype of A7445G, CC genotype of C7711T, AG genotype of G17937A, and GG genotype of G20937A had superior growth and meat quality traits compared to individuals with other genotypes. Furthermore, we 


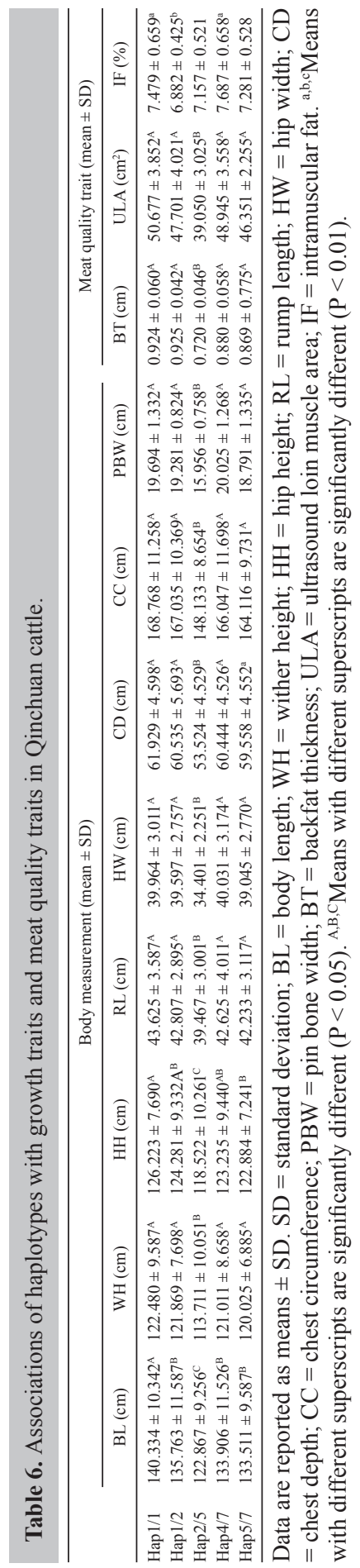


also examined the association of haplotype combinations with some traits, including BL, RL, $\mathrm{CC}, \mathrm{CD}, \mathrm{PBW}, \mathrm{ULA}$, and IF. The proportion of individuals with diplotypes $\mathrm{H}_{1} \mathrm{H}_{1}$ (AA-CCGG-CC), $\mathrm{H}_{1} \mathrm{H}_{2}$ (AA-CC-GG-CG), and $\mathrm{H}_{4} \mathrm{H}_{7}$ (AG-TT-GG-CC) was higher than that of other haplotype combinations. The high-frequency haplotypes have most likely been present in the population for a long time and may be regulated directly or indirectly by different rearing environments ( $\mathrm{Li}$ et al., 2013). Individuals with the $\mathrm{H}_{1} \mathrm{H}_{1}$ haplotype showed superior growth and meat quality traits such as BT, BL, HH, ULA, and IF.

In addition, the $\mathrm{A} 7445 \mathrm{G}$ mutation was intronic and $\mathrm{C} 7711 \mathrm{~T}$ was a synonymous mutation, and these two mutations did not change the structure of their encoded proteins. Earlier reports concluded that SNPs resulting in synonymous mutations can affect gene expression, phenotype, and, consequently, respective physiological functions (Van Laere et al., 2003; Krawczak et al., 2007). Therefore, these two variations may influence mitochondrial morphology and adipogenesis by affecting the transcription of the SIRT2 gene in cattle. Further verification is needed to elucidate the underlying mechanism.

In summary, we reported four polymorphisms of the SIRT2 gene in Qinchuan cattle and determined the association of the SIRT2 gene with BMTs and meat quality traits. The present data suggest that the individuals of the combined genotype $\mathrm{H}_{1} \mathrm{H}_{1}$ have superior growth and meat quality traits. Our investigation provides evidence that the SIRT2 gene could be used as a candidate gene for cattle breeding. Further research should be conducted in a large population before applying this gene to molecular marker-assisted selection.

\section{ACKNOWLEDGMENTS}

Research supported by the National "Five Year" Science and Technology Support Project (\#2011BAD28B04-03), the China National "863" Program (\#2011AA100307), the GMO New Varieties Major Project (\#2011ZX08007-002), the National Beef and Yak Industrial Technology System (\#CARS-38), the "13115" Scientific and Technological Innovation Program of Shaanxi Province, the Qinchuan Beef Cattle Breeding High-Quality and Efficient Breed Breeding Technology Research Extension Project (\#2011KTCL02-07), the Chinese Beef Cattle Economically Important Traits Functional Genomics Studies Project (2013AA102505), and the Identification and Regulation of Qinchuan Cattle Meat Quality Traits Functional Gene Project (\#31272411).

\section{REFERENCES}

Ardlie KG, Kruglyak L and Seielstad M (2002). Patterns of linkage disequilibrium in the human genome. Nat. Rev. Genet. 3: 299-309.

Barrett JC, Fry B, Maller J and Daly MJ (2005). Haploview: analysis and visualization of LD and haplotype maps. Bioinformatics 21: 263-265.

Blander G and Guarente L (2004). The Sir2 family of protein deacetylases. Annu. Rev. Biochem. 73: 417-435.

Dryden SC, Nahhas FA, Nowak JE, Goustin AS, et al. (2003). Role for human SIRT2 NAD-dependent deacetylase activity in control of mitotic exit in the cell cycle. Mol. Cell Biol. 23: 3173-3185.

Fan YY, Zan LS, Fu CZ, Tian WQ, et al. (2011). Three novel SNPs in the coding region of PPAR $\gamma$ gene and their associations with meat quality traits in cattle. Mol. Biol. Rep. 38: 131-137.

Gilbert RP, Bailey DR and Shannon NH (1993). Linear body measurements of cattle before and after 20 years of selection for postweaning gain when fed two different diets. J. Anim. Sci. 71: 1712-1720.

Guarente L (2007). Sirtuins in aging and disease. Cold Spring Harb. Symp. Quant. Biol. 72: 483-488.

Jiao Y, Zan LS, Liu YF, Wang HB, et al. (2010). A novel polymorphism of the MYPN gene and its association with meat 
quality traits in Bos taurus. Genet. Mol. Res. 9: 1751-1758.

Jing E, Gesta S and Kahn CR (2007). SIRT2 regulates adipocyte differentiation through FoxO1 acetylation/deacetylation. Cell Metab. 6: 105-114.

Krawczak M, Thomas NS, Hundrieser B, Mort M, et al. (2007). Single base-pair substitutions in exon-intron junctions of human genes: nature, distribution, and consequences for mRNA splicing. Hum. Mutat. 28: 150-158.

Krishnan J, Danzer C, Simka T, Ukropec J, et al. (2012). Dietary obesity-associated Hiflalpha activation in adipocytes restricts fatty acid oxidation and energy expenditure via suppression of the Sirt2-NAD+ system. Genes Dev. 26: 259-270.

Li M, Sun X, Hua L, Lai X, et al. (2013). SIRT1 gene polymorphisms are associated with growth traits in Nanyang cattle. Mol. Cell. Probes 27: 215-220.

Liu Y, Zan L, Zhao S, Xin Y, et al. (2010). Molecular characterization, polymorphism of bovine ZBTB38 gene and association with body measurement traits in native Chinese cattle breeds. Mol. Biol. Rep. 37: 4041-4049.

Marmorstein R (2004). Structure and chemistry of the Sir2 family of NAD+-dependent histone/protein deactylases. Biochem. Soc. Trans. 32: 904-909.

Nahhas F, Dryden SC, Abrams J and Tainsky MA (2007). Mutations in SIRT2 deacetylase which regulate enzymatic activity but not its interaction with HDAC6 and tubulin. Mol. Cell. Biochem. 303: 221-230.

Nei M and Roychoudhury AK (1974). Sampling variances of heterozygosity and genetic distance. Genetics 76: 379-390.

North BJ and Verdin E (2007). Mitotic regulation of SIRT2 by cyclin-dependent kinase 1-dependent phosphorylation. $J$. Biol. Chem. 282: 19546-19555.

North BJ, Marshall BL, Borra MT, Denu JM, et al. (2003). The human Sir2 ortholog, SIRT2, is an NAD+-dependent tubulin deacetylase. Mol. Cell 11: 437-444.

Peck B, Chen CY, Ho KK, Di Fruscia P, et al. (2010). SIRT inhibitors induce cell death and p53 acetylation through targeting both SIRT1 and SIRT2. Mol. Cancer Ther. 9: 844-855.

Rincon G, Farber EA, Farber CR, Nkrumah JD, et al. (2009). Polymorphisms in the STAT6 gene and their association with carcass traits in feedlot cattle. Anim. Genet. 40: 878-882.

Sambrook J and Russell DW (2001). Molecular Cloning: a Laboratory Manual. Cold Spring Harbor Laboratory Press, Cold Spring Harbor.

Tian WQ, Wang HC, Song FB, Zan LS, et al. (2011). Association between a single nucleotide polymorphism in the bovine chemerin gene and carcass traits in Qinchuan cattle. Genet. Mol. Res. 10: 2833-2840.

Van Laere AS, Nguyen M, Braunschweig M, Nezer C, et al. (2003). A regulatory mutation in IGF2 causes a major QTL effect on muscle growth in the pig. Nature 425: 832-836.

Wang F and Tong Q (2009). SIRT2 suppresses adipocyte differentiation by deacetylating FOXO1 and enhancing FOXO1's repressive interaction with PPAR $\gamma$. Mol. Biol. Cell 20: 801-808.

Xue M, Zan LS, Gao L and Wang HB (2011). A novel polymorphism of the myogenin gene is associated with body measurement traits in native Chinese breeds. Genet. Mol. Res. 10: 2721-2728.

Zhou Y, Zhang H, He B, Du J, et al. (2012). The bicyclic intermediate structure provides insights into the desuccinylation mechanism of human sirtuin 5 (SIRT5). J. Biol. Chem. 287: 28307-28314. 